\title{
AN EQUIVALENCE BETWEEN NONASSOCIATIVE RING THEORY AND THE THEORY OF A SPECIAL CLASS OF GROUPS
}

\author{
KENNETH W. WESTON
}

Introduction. A. Malcev constructed $[1$, p. 221] an interesting correspondence $\mathrm{X}$ between the class $\Re_{1}$ of all nonassociative rings with identity and a certain class of groups $G_{5}$, nilpotent of class at most 2. Malcev proved [ 1 , Theorem 1, p. 226] by means of this correspondence that the theories of $\Re_{1}$ and $G_{5}$ are equivalent. This theorem is generalized here by showing that all of nonassociative ring theory is equivalent to the theory of a larger class of groups (5).

We prove specifically

THEOREM 1. (a) There is a one to one mapping $F$ of the class of all nonassociative rings $\Re$ onto a class of groups (H) where (5) is defined by

$G \in\left(3 \Leftrightarrow \mathrm{A}_{1} . G\right.$ is nilpotent of class at most 2 (i.e. $G_{3}=1, G_{3}$ is the third term of the lower central series).

$\Leftrightarrow \mathrm{A}_{2}$. G has 2 operators: $\alpha, \beta$ where $\alpha^{2}=\beta^{2}=\alpha \beta=\beta \alpha=0$.

$\Leftrightarrow \mathrm{A}_{3}$. If $K_{\alpha}$ and $K_{\beta}$ are the kernels of $\alpha$ and $\beta$ respectively then $K_{\alpha} \cap K_{\beta} \subseteq Z(G) .(Z(G)$ is the center of $G$.)

$\Leftrightarrow \mathrm{A}_{4} . K_{\alpha}$ and $K_{\beta}$ are abelian.

$\Leftrightarrow \mathrm{A}_{5}$. There are 2 homomorphisms $\tilde{\alpha}$ and $\tilde{\beta}$ defined on $K_{\alpha} \cap K_{\beta} \rightarrow G$ satisfying $X^{\tilde{\alpha} \alpha}=X^{\tilde{\beta} \beta}=X$ and $X^{\tilde{\alpha} \beta}=X^{\tilde{\beta} \alpha}=1$, $X \in K_{\alpha} \cap K_{\beta}$.

(b) If $T_{\Re}$ and $T_{\&}$ denote standard formalization of the theories for $\Re$ and (s) respectively in the sense of Tarski-Mostowski-Robinson, Undecidable theories [2, I.2] then $F$ induces a one to one recursive mapping $\bar{F}$ of all the closed formulas in $T_{\Re}$ onto those of $T_{\mathbb{B}}$. Also if $R \in \Re$ and $P$ is a closed formula of $T_{\Re}$ then $P$ is true in $R$ if and only if $\bar{F}(P)$ is true in $F(R)$. Likewise $F^{-1}$ induces a one to one recursive mapping $\bar{F}^{-1}$ of all the closed formulas in $T \&$ onto those of $T_{\Re}$ where $G \in(B)$ and $Q$ is a closed formula of $T$ es implies that $Q$ is true in $G$ if and only if $\bar{F}^{-1}(Q)$ is true in $F^{-1}(G)$.

(c) $F\left(\Re_{1}\right)=G_{5}$

The axioms: $A_{1}, \cdots, A_{5}$ are not in their weakest form.

EXAMPLE. It can be shown that $A_{4}$ can be replaced by the assumption that either $K_{\alpha}$ or $K_{\beta}$ is abelian.

Received by the editors June 7, 1967 and, in revised form, July 27, 1967. 
Proof of Theorem 1 (a). If $R \in \Re$ denote by $F(R)$ the collection of unipotent matrices

$$
\left(\begin{array}{lll}
1 & y & z \\
0 & 1 & x \\
0 & 0 & 1
\end{array}\right), \quad x, y, z \in R
$$

It is perfectly straightforward to show that $F(R)$ forms a group with identity

$$
\left(\begin{array}{lll}
1 & 0 & 0 \\
0 & 1 & 0 \\
0 & 0 & 1
\end{array}\right)
$$

and

$$
\left.\left\{\begin{array}{lll}
1 & 0 & x \\
0 & 1 & 0 \\
0 & 0 & 1
\end{array}\right\}, x \in R\right\} \subseteq Z(F(R))
$$

We shall first verify axioms: $A_{1}, \cdots, A_{5}$. In order to do so, assume that $g$ and $h$ are elements of a group $G$ and define the group commutator of $g$ and $h$ to be the product $g^{-1} \cdot h^{-1} \cdot g \cdot h$. If we denote the commutator of $g$ and $h$ by $[g, h]$, then direct calculation yields

$$
\left[\left(\begin{array}{lll}
1 & b & c \\
0 & 1 & a \\
0 & 0 & 1
\end{array}\right),\left(\begin{array}{lll}
1 & y & z \\
0 & 1 & x \\
0 & 0 & 1
\end{array}\right)\right]=\left(\begin{array}{ccc}
1 & 0 & b x-y a \\
0 & 1 & 0 \\
0 & 0 & 1
\end{array}\right), a, b, c, x, y, z \in R
$$

Therefore by (1) and (2), $F(R)$ is nilpotent of class at most 2 and axiom $A_{1}$ is satisfied.

Next define the mappings $\alpha$ and $\beta$ on $F(R)$ by

$$
\begin{aligned}
& \left(\begin{array}{lll}
1 & y & z \\
0 & 1 & x \\
0 & 0 & 1
\end{array}\right)^{\alpha}=\left(\begin{array}{rrr}
1 & 0 & -y \\
0 & 1 & 0 \\
0 & 0 & 1
\end{array}\right), \\
& \left(\begin{array}{lll}
1 & y & z \\
0 & 1 & x \\
0 & 0 & 1
\end{array}\right)^{\beta}=\left(\begin{array}{lll}
1 & 0 & x \\
0 & 1 & 0 \\
0 & 0 & 1
\end{array}\right), \quad x, y, z \in R .
\end{aligned}
$$

It's trivial to show that $\alpha$ and $\beta$ are operators over $F(R)$ and $\alpha^{2}=\beta^{2}$ $=\alpha \beta=\beta \alpha=0$. Thus $A_{2}$ is satisfied. 
If $K_{\alpha}$ and $K_{\beta}$ are the kernels of $\alpha$ and $\beta$ respectively then

(4) $K_{\alpha}=\left\{\left(\begin{array}{lll}1 & 0 & y \\ 0 & 1 & x \\ 0 & 0 & 1\end{array}\right), x, y \in R\right\}, \quad K_{\beta}=\left\{\left(\begin{array}{lll}1 & x & y \\ 0 & 1 & 0 \\ 0 & 0 & 1\end{array}\right), x, y \in R\right\}$.

Hence by (1)

$$
K_{\alpha} \cap K_{\beta}=\left\{\left(\begin{array}{lll}
1 & 0 & x \\
0 & 1 & 0 \\
0 & 0 & 1
\end{array}\right), x \in R\right\} \subseteq Z(F(R)) .
$$

That $K_{\alpha}$ and $K_{\beta}$ are abelian follows directly from (4).

Using (5) we can define mappings $\tilde{\alpha}$ and $\tilde{\beta}$ on $K_{\alpha} \cap K_{\beta} \rightarrow G$ by

(6) $\left(\begin{array}{lll}1 & 0 & x \\ 0 & 1 & 0 \\ 0 & 0 & 1\end{array}\right)^{\tilde{\alpha}}=\left(\begin{array}{rrr}1 & -x & 0 \\ 0 & 1 & 0 \\ 0 & 0 & 1\end{array}\right),\left(\begin{array}{lll}1 & 0 & x \\ 0 & 1 & 0 \\ 0 & 0 & 1\end{array}\right)^{\tilde{\beta}}=\left(\begin{array}{lll}1 & 0 & 0 \\ 0 & 1 & x \\ 0 & 0 & 1\end{array}\right), x \in R$.

Direct calculation shows that $\tilde{\alpha}$ and $\tilde{\beta}$ are homomorphisms of $K_{\alpha} \cap K_{\beta}$ into $K_{\beta}$ and $K_{\alpha}$ respectively and

$$
\begin{aligned}
& \left(\begin{array}{lll}
1 & 0 & x \\
0 & 1 & 0 \\
0 & 0 & 1
\end{array}\right)^{\tilde{\alpha} \alpha}=\left(\begin{array}{lll}
1 & 0 & x \\
0 & 1 & 0 \\
0 & 0 & 1
\end{array}\right)^{\tilde{\beta} \beta}=\left(\begin{array}{lll}
1 & 0 & x \\
0 & 1 & 0 \\
0 & 0 & 1
\end{array}\right), \\
& \left(\begin{array}{lll}
1 & 0 & x \\
0 & 1 & 0 \\
0 & 0 & 1
\end{array}\right)^{\tilde{\alpha} \beta}=\left(\begin{array}{lll}
1 & 0 & x \\
0 & 1 & 0 \\
0 & 0 & 1
\end{array}\right)^{\tilde{\beta} \alpha}=\left(\begin{array}{lll}
1 & 0 & 0 \\
0 & 1 & 0 \\
0 & 0 & 1
\end{array}\right) .
\end{aligned}
$$

We have shown thus far that $F(R)$ satisfies axioms: $A_{1}, \cdots, A_{5}$. Now we determine a mapping $H:(\mathfrak{B} \rightarrow \Re$ such that

$$
\begin{aligned}
& \text { (a) } \quad F(H(G)) \cong G, \quad G \in(\xi) \\
& \text { (b) } H(F(R)) \cong R, \quad R \in \Re
\end{aligned}
$$

and hence $F$ is one to one and onto.

For $G \in(B)$ let $H(G)=K_{\alpha} \cap K_{\beta}$ where addition $\oplus$ and multiplication $\otimes$ are defined by

(8) $\quad g_{1} \oplus g_{2}=g_{1} \cdot g_{2}, \quad g_{1} \otimes g_{2}=\left[\begin{array}{cc}\tilde{\beta} & \tilde{\alpha} \\ g_{2}, & g_{1}\end{array}\right], \quad g_{1}, g_{2} \in H(G)$.

Obviously $H(G)$ forms an abelian group under addition. $H(G)$ is also closed under multiplication since 


$$
\left(g_{1} \otimes g_{2}\right)^{\alpha}=\left[\begin{array}{cc}
\tilde{\beta} & \tilde{\alpha} \\
g_{2}, & g_{1}^{\alpha}
\end{array}\right]^{\tilde{\beta}}=\left[\begin{array}{cc}
\tilde{\beta} \alpha & \tilde{\alpha} \alpha \\
g_{2} & g_{1}
\end{array}\right]=\left[1, g_{1}\right]=1
$$

and

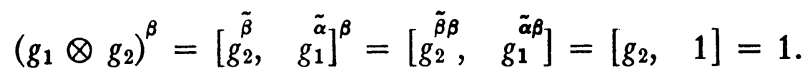

The distributive laws also follow.

To prove 7 (b) let $R \in \Re$. By (5)

$$
H(F(R))=\left\{\left(\begin{array}{lll}
1 & 0 & x \\
0 & 1 & 0 \\
0 & 0 & 1
\end{array}\right), \quad x \in R\right\} .
$$

Also, by (8), (6) and (2)

$$
\begin{aligned}
\left(\begin{array}{lll}
1 & 0 & x \\
0 & 1 & 0 \\
0 & 0 & 1
\end{array}\right) \oplus\left(\begin{array}{lll}
1 & 0 & y \\
0 & 1 & 0 \\
0 & 0 & 1
\end{array}\right) & =\left(\begin{array}{lll}
1 & 0 & x+y \\
0 & 1 & 0 \\
0 & 0 & 1
\end{array}\right), \\
\left(\begin{array}{lll}
1 & 0 & x \\
0 & 1 & 0 \\
0 & 0 & 1
\end{array}\right) \otimes\left(\begin{array}{lll}
1 & 0 & y \\
0 & 1 & 0 \\
0 & 0 & 1
\end{array}\right) & =\left[\left(\begin{array}{lll}
1 & 0 & y \\
0 & 1 & 0 \\
0 & 0 & 1
\end{array}\right),\left(\begin{array}{lll}
1 & 0 & x \\
0 & 1 & 0 \\
0 & 0 & 1
\end{array}\right)\right] \\
& =\left[\left(\begin{array}{lll}
1 & 0 & 0 \\
0 & 1 & y \\
0 & 0 & 1
\end{array}\right),\left(\begin{array}{ccc}
1 & -x & 0 \\
0 & 1 & 0 \\
0 & 0 & 1
\end{array}\right)\right] \\
& =\left(\begin{array}{lll}
1 & 0 & x \cdot y \\
0 & 1 & 0 \\
0 & 0 & 1
\end{array}\right) .
\end{aligned}
$$

To prove 7 (a) is somewhat more difficult. Assume that $G \in(B)$ and $X \in F(H(G))$, i.e.

$$
X=\left(\begin{array}{ccc}
1 & h_{2} & h_{3} \\
0 & 1 & h_{1} \\
0 & 0 & 1
\end{array}\right), \quad h_{1}, h_{2}, h_{3} \in K_{\alpha} \cap K_{\beta} .
$$

Define a mapping $\tau$ on $F(H(G)) \rightarrow G$ by

$$
X^{\tau}=h_{1}^{\tilde{\beta}}\left(h_{2}^{\tilde{\alpha}}\right)^{-1} h_{3} \text {. }
$$

In order to first show that $\tau$ is a homomorphism let 


$$
Y=\left(\begin{array}{ccc}
1 & k_{2} & k_{3} \\
0 & 1 & k_{1} \\
0 & 0 & 1
\end{array}\right), \quad k_{1}, k_{2}, k_{3} \in K_{\alpha} \cap K_{\beta},
$$

and apply (8) and (9) to get

$$
\begin{aligned}
(X \cdot Y)^{\tau} & =\left(\begin{array}{ccc}
1 & h_{2} \oplus k_{2} & \left(h_{2} \otimes k_{1}\right) \oplus h_{3} \oplus k_{3} \\
0 & 1 & h_{1} \oplus k_{1} \\
0 & 0 & 1
\end{array}\right)^{\tau} \\
& =\left(h_{1} k_{1}\right)^{\tilde{\beta}}\left(k_{2}^{-1}\right)^{\tilde{\alpha}}\left(h_{2}^{-1}\right)^{\tilde{\alpha}}\left[k_{1}^{\tilde{\beta}}, h_{2}^{\tilde{\alpha}}\right] h_{3} k_{3} .
\end{aligned}
$$

Since

$$
\left(Y^{\tau}\right)^{-1}=k_{3}^{-1} k_{2}^{\tilde{\alpha}}\left(k_{1}^{-1}\right)^{\tilde{\beta}},
$$

by axioms $A_{1}, A_{3}$ and $A_{4}$ we have

$$
(X \cdot Y)^{\tau}\left(Y^{\tau}\right)^{-1}=\left(h_{1} k_{1}\right)^{\tilde{\beta}}\left(h_{2}^{-1}\right)^{\tilde{\alpha}}\left(k_{1}^{-1}\right)^{\tilde{\beta}}\left[k_{1}^{\tilde{\beta}}, h_{2}^{\tilde{\alpha}}\right] h_{3} .
$$

Now every nilpotent group $N$ of class at most 2 satisfies the identity $\left[u^{-1}, v^{-1}\right]=[u, v], u, v \in N$ (i.e. observe that

$$
1=\left[u, v \cdot v^{-1}\right]=[u, v] \cdot\left[u, v^{-1}\right],
$$

which in turn implies $\left.[u, v]^{-1}=\left[u, v^{-1}\right]\right)$. Consequently

$$
\left[\tilde{k_{1}}, h_{2}^{\tilde{\alpha}}\right]=k_{1}^{\tilde{\beta}} h_{2}^{\tilde{\alpha}}\left(k_{1}^{\tilde{\beta}}\right)^{-1}\left(h_{2}^{\tilde{\alpha}}\right)^{-1} \text {. }
$$

By (10) and (11) we can see then

$$
(X \cdot Y)^{\tau}\left(Y^{\tau}\right)^{-1}=h_{1}^{\tilde{\beta}}\left(h_{2}^{\tilde{\alpha}}\right)^{-1} h_{3}=X^{\tau} .
$$

Hence $\tau$ is a homomorphism.

We wish to show now that the kernel of $\tau$ is the identity. Therefore let

$$
X=\left(\begin{array}{ccc}
1 & h_{2} & h_{3} \\
0 & 1 & h_{1} \\
0 & 0 & 1
\end{array}\right), \quad h_{1}, h_{2}, h_{3} \in K_{\alpha} \cap K_{\beta},
$$

and

$$
X^{\tau}=h_{1}^{\tilde{\beta}}\left(h_{2}^{\tilde{\alpha}}\right)^{-1} h_{3}=1 .
$$

Thus $h_{1}^{\tilde{\beta} \beta}=\left(h_{3}^{\beta}\right)^{-1}\left(h_{2}^{\tilde{\alpha}}\right)^{\beta}$ and by $A_{5}$ 


$$
h_{1}=\left(h_{3}^{\beta}\right)^{-1}\left(h_{2}^{\tilde{\alpha}}\right)^{\beta}=1 .
$$

Likewise

$$
\left(h_{1}^{\tilde{\beta}}\right)^{\alpha}=\left(h_{3}^{\alpha}\right)^{-1}\left(h_{2}^{\tilde{\alpha}}\right)^{\alpha}, \quad 1=h_{2} .
$$

But $h_{1}=h_{2}=1$ implies $h_{3}=1$ and hence $X$ is the identity.

To show that $\tau$ is an onto mapping let $g \in G$ and $g_{1}=g^{\alpha}, g_{2}=g^{\beta}$ and $g_{3}=\left(g_{2}^{\tilde{\beta}}\right)^{-1} g\left(g_{1}^{\tilde{\alpha}}\right)^{-1}$. Consequently (by $\left.A_{2}\right) g_{1}, g_{2}, g_{3} \in K_{\alpha} \cap H_{\beta}$ and

$$
X=\left(\begin{array}{ccc}
1 & g_{1}^{-1} & g_{3} \\
0 & 1 & g_{2} \\
0 & 0 & 1
\end{array}\right) \in F(H(G)) .
$$

Thereforeby $A_{3}, X^{\tau}=g_{2}^{\tilde{\beta}} g_{1}^{\tilde{\alpha}} g_{3}=g_{2}^{\tilde{\beta}} g_{3} g_{1}^{\tilde{\alpha}}=g$. Thus $H$ is the inverse map of $F$.

Proof of THEOREM 1 (b). We assume that the logical symbolism of $T_{\Re}$ and $T_{\Theta}$ is that of [2]. Suppose also that $T_{\Re}$ contains among its list of primitive symbols: $\mathbf{X},+, 0$, denoting: multiplication, addition and the additive identity respectively. Let $T_{\infty}$ contain among its primitive symbols: $\tilde{\boldsymbol{\alpha}}(), \tilde{\underline{\beta}}(),[],, \cdot, 1$ to denote: homomorphisms: $\tilde{\alpha}, \widetilde{\beta}$, commutation, multiplication and identity respectively. Suppose also $K$ denotes a formula in $T_{\Theta}$ representing the predicate $x \in K_{\alpha} \cap K_{\beta}$.

Given a formula $P$ in $T_{\Re}$ we construct a new formula $B$ of $T_{B}$ by replacing each occurrence of: $x_{i} \times x_{j}, x_{i}+x_{j}$ and 0 in $P$ by: $\left[\tilde{\beta}\left(x_{j}\right), \tilde{\alpha}\left(x_{i}\right)\right]$, $x_{i} \cdot x_{j}, 1$ respectively. Let $B^{K}$ denote the formula obtained by relativizing $B$ to $K$, [2, p. 25]. The map $\bar{F}$ is defined by $\bar{F}(P)=B^{K}$. It's clear from the construction of $H$ that $P$ is true in $R$ if and only if $B^{K}$ is true in $F(R)$.

For the converse assume that $P$ is a formula in $T_{\leftrightarrow}$. Transform $P$ recursively into prenex form [3, Theorem 19, p. 167] $Q_{1} x_{1}, \cdots$, $Q_{n} x_{n} S\left(x_{1}, \cdots, x_{n}, 1\right)$ where $Q_{i}$ represents a quantifier and $S\left(x_{1}, \cdots, x_{n}, 1\right)$ is a formula in $T_{\mathscr{S}}$ with all of its variables: $x_{1}, \cdots$, $x_{n}$, occurring free. We may assume without generality that each variable $x_{i}$ occurs with positive exponent. From this we construct a formula $C$ in $T_{\Re}$ defined by

$$
Q_{1} x_{1} Q_{1} y_{1} Q_{1} z_{1}, \cdots, Q_{n} x_{n} Q_{n} y_{n} Q_{n} z_{n} S^{\prime}\left(x_{1}, y_{1}, z_{1}, \cdots, x_{n}, y_{n}, z_{n}, 0\right)
$$

where $S^{\prime}\left(x_{1}, y_{1}, z_{1}, \cdots, x_{n}, y_{n}, z_{n}, 0\right)$ results from $S\left(x_{1}, \cdots, x_{n}, 1\right)$ by replacing every equation of the form: $x_{i_{1}} \cdots x_{i_{k}}=1$ by

$$
\begin{aligned}
\left(x_{i_{1}}+x_{i_{2}}+\cdots+x_{i_{k}}=0\right) \wedge & \left(y_{i_{1}}+y_{i_{2}}+\cdots+y_{i_{k}}=0\right) \\
\wedge & \left(\left(y_{i_{1}}+y_{i_{2}}+\cdots+y_{i_{k-1}}\right) x_{i_{k}}\right. \\
& +\left(y_{i_{1}}+\cdots+y_{i_{k-2}}\right) x_{i_{k-1}}+\cdots \\
& \left.+y_{i_{1}} x_{i_{2}}+z_{i_{1}}+\cdots+z_{i_{k}}=0\right)
\end{aligned}
$$


and every equation of the form: $x_{j_{1}} x_{j_{2}} \cdots x_{j_{m}}=x_{l_{1}} x_{l_{q}} \cdots x_{l_{q}}$ by

$$
\begin{aligned}
& \left(x_{j_{1}}+x_{j_{2}}+\cdots+x_{j_{m}}=x_{l_{1}}+x_{l_{2}}+\cdots+x_{l_{q}}\right) \\
& \wedge\left(y_{j_{1}}+y_{j_{2}}+\cdots+y_{j_{m}}=y_{l_{1}}+y_{l_{2}}+\cdots+y_{l_{q}}\right) \\
& \wedge\left(y_{j_{1}}+y_{j_{2}}+\cdots+y_{j_{m-1}}\right) x_{j_{m}} \\
& \quad+\left(y_{j_{1}}+y_{j_{2}}+\cdots+y_{j_{m-q}}\right) x_{j_{m-1}}+\cdots+y_{j_{1}} x_{j_{2}}+z_{j_{1}}+\cdots+z_{j_{m}} \\
& \quad=\left(y_{l_{1}}+y_{l_{2}}+\cdots+y_{l_{q_{-}}}\right) x_{l_{q}}+\left(y_{l_{1}}+y_{l_{2}}+\cdots+y_{l_{-2}}\right) x_{l_{q-1}} \\
& \left.\quad+\cdots+y_{l_{1}} x_{l_{2}}+z_{l_{1}}+\cdots+z_{l_{m}}\right) .
\end{aligned}
$$

Define the map $\bar{F}^{-1}$ by $\bar{F}^{-1}(P)=C$. As is obvious from the construction of $F, P$ is true in $G$ if and only if $C$ is true in $F^{-1}(G)$.

Proof of Theorem 1 (c). The correspondence $X$ in $[1]$ is the following.

If $R \in \Re_{1}$ let $\mathbf{X}(R)$ denote the collection of ordered triples $\left(x_{1}, x_{2}, x_{3}\right)$ of elements from $R$ and define multiplication by

$$
\left(x_{1}, x_{2}, x_{3}\right) \cdot\left(y_{1}, y_{2}, y_{3}\right)=\left(x_{1}+y_{1}, x_{2}+y_{2}, x_{2} \cdot y_{1}+x_{3}+y_{3}\right) .
$$

One has only to make the correspondence

$$
\left(\begin{array}{lll}
1 & x_{2} & x_{3} \\
0 & 1 & x_{1} \\
0 & 0 & 1
\end{array}\right) \leftrightarrow\left(x_{1}, x_{2}, x_{3}\right)
$$

between $F(R)$ and $\mathrm{X}(R)$ to see $F(R) \cong \mathrm{X}(R)$. Since $G_{5}=\mathrm{X}\left(\Re_{1}\right)$, the proof is complete.

The proof of the following statement follows from (2).

TheOREM 2. The operators $\alpha$ and $\beta$ in $A_{2}$ are left commutation by matrices:

$$
\left(\begin{array}{lll}
1 & 0 & 0 \\
0 & 1 & 1 \\
0 & 0 & 1
\end{array}\right) \text { and }\left(\begin{array}{lll}
1 & 1 & 0 \\
0 & 1 & 0 \\
0 & 0 & 1
\end{array}\right)
$$

respectively.

\section{REFERENCES}

1. A. Malcev, On a correspondence between rings and groups, Amer. Math. Soc. Transl. (2) 45 (1960), 221-231.

2. A. Tarski, A. Mostowski and R. Robinson, Undecidable theories, NorthHolland, Amsterdam, 1953.

3. S. Kleene, Introduction to metamathematics, Van Nostrand, Princeton, N. J., 1952.

University of Notre DAme 